\title{
On the Conductivity of Perfusion Fluid of Frog's Heart under Abnormal Atmospheric Pressure.
}

\author{
(According to the experiment of H. Miyazaki* and M. Ogata)
}

By

Sakuzi Kodama.

（小王作治）

(From the Institute of Physiology, Director: Prof. S. Kodama, Kumamoto Medical College, Kumamoto.)

Abnormal atmospheric pressure exerts mechanical influence not only upon the surface, but also upon the interior of body. All the tissue cells of the body exist thus under the abnormal pressure. Physicochemical conditions and properties of the body fluid and also cells have perhaps been developed under the present atmospheric condition as a factor at least. Physicochemical study of the body fluid under abnormal atmospheric pressure may be an important branch of the high and low pressure physiology. So far as we know, reports on this line are very scanty, and even those can not be regarded as such as we expect, and shall not be here referred as our control. Some chemical changes have been noticed by a few investigators in the blood of the animal under abnormal atmospheric pressure.

B ornste i ${ }^{1)}$ found hydraemia of dog and monkey under high atmospheric pressure (2 atmosph.). His finding has been very often referred to by later investigators, but they disregard the fact that the experiment of Born ste in was of longer duration (several months) and could not directly be compared with those of short duration (a few hours). S a kai ${ }^{2)}$ found an increase of viscosity and serum protein of Caisson worker by repetition of work, which he regarded as a cause of adaptation of the worker to the high pressure. Mot e $\mathbf{~}^{31}$ says that he

* The essentials of the results on the high pressure were read by H. Miy a $\mathrm{zaki}$ before the annual meeting of the Kumamoto Medical Society, November, 1937.

1) Bornstein, A., Pflügers Arch., 1911, 138, 609.

2) Sa k a i, Y., Nippon Naikagakkai Z., 1927, 15, 575.

3) Moteki, K., Kaigun-gun-i-kai Z., 1931, 20, 428. 
found decrease of serum protein and a tendency of increase of blood chloride of rabbit under $30 \mathrm{~mm} \mathrm{Hg}$. pressure increase for one hour. Ka gi i a $\mathrm{m} \mathrm{a}^{4556)}$ performed a more systematic experiment on the high pressure (15-30 pounds), and found some chemical changes of blood of rabbit and man, decrease of protein and chloride of the serum and also of viscosity. H o s o ka w a ${ }^{7}$ attributed a decrease of haemoglobin under high pressure ( $30-50$ pounds) to the hydraemia which he believed to be an established fact. He found further that the resistance of blood corpuscle to hypotonic solution weakened under high pressure, but this was also the case under low atmospheric pressure. Shilling, Thomson, Behnke, Shaw and Messer ${ }^{3)}$ did not prove the chemical change of the blood of dog under 45 pounds of $97 \%$ oxygen during $2-4$ hours.

On chemical changes of blood of animal and man under low atmospheric pressure, references available here are still few. End ${ }^{9 /}$ reported that blood quantity and protein decreased under low atmospheric pressure at first and recovered later, and chloride and non-protein nitrogen increased at first and recovered later. These findings are not the reverse of those under high atmospheric pressure. Loe $\mathrm{y}^{10)}$ perceived decrease of calcium of blood at high mountain and slowing of the sedimentation velocity, which he was inclined to attribute to change of plasma protein. Sekiguti ${ }^{(1)}$ observed a tendency of increase of serum protein and colloid-osmotic pressure of serum, but his data showed a too large variation for any definite decision.

Our experiment to be reported in this paper was as the first intended as a study of the influence of abnormal atmospheric pressure upon the physicochemical properties of the blood.

\section{Method.}

As a pressure chamber an iron box was used which was in general of the same structure as that described in a previous paper ${ }^{12)}$ from our institute, but which was larger to allow a small kymograph to be put in.

Heart of rana nigromaculata was employed for the experiment. Stra u b's perfusion preparation of heart was made, the fluid reservoir of the cannula being larger enough to serve as a conductivity vessel with

4) Ka g i y a m a, S., Kumamoto Igakkai Z., 1934, 10, 263.

5) Kagi i a m a, S., ibid., 519.

6) Kag i y a ma, S., ibid., 528.

7) Ho s o kawa, S., Kaigun-gun-i-kai Z., 1936, 25, 73.

8) Shilling, C. W., R.M. Thomson, A. R. Behnke, L. A. Shaw and A. C. Messer, Am. J. Physiol., 1934, 107, 29.

9) En d o, H., Kaigun-gun-i-kai Z., 1934, 23, 405.

10) L oew 8, A., Kl. W., 1934, 13, 545.

11) Sekiguti, E., Kaigun-gun-i-kai Z., 1936, 25, 633.

12) Kod a ma, S., Tohoku J. Exp. Med., 1937, 31, 357. 
ca. 4 cc content. Two cores connected with two poles of the conductivity vessel were led out through a side hole of the pressure box to a Kohlrausch's bridge which was used to measure the conductivity of the perfusion fluid in the vessel. Kohlrausch's arrangement as in the usual manner consisted of a rheocord, resistance boxes, a small inductorium and a telephone. As the experiment was made at room temperature which was not kept at constant, the specific conductivity of the perfusion fluid at each temperature had to be calculated for a standard temperature to be compared to each other and to be used for calculation of the mean value. In the case of high pressure experiment, $28^{\circ}$ was taken as the standard temperature and the specific conductivity of the perfusion fluid at each temperature was recalculated for that temperature. This was done as follows: The difference between the rheocord reading (the resistance box being always at constant) of perfusion fluid before pressure increase, after the preparation had reached an equilibrium state, and those during the pressure experiment was taken, which was added to the rheocord reading before pressure increase at $28^{\circ}$. Allowance was made in this case for the fact that the change of the rheocord reading during the pressure experiment from that before pressure increase was the same at each temperature: this change was, strictly taken, not the same, but might be small during so small a temperature range (ca. $3^{\circ}$ ).

In the case of low pressure experiment, the vessel constant of conductivity was previously measured at various temperatures, which was applied for the calculation of the conductivity of the perfusion fluid at respective temperatures. Recalculation for a standard temperature was not made in this case.

Change of conductivity was always expressed by the ratio of the value under abnormal pressure before pressure change.

High pressure in the chamber was attained by an air compressor and regulated at a constant level to \pm one pound by a specially devised apparatus. Low atmospheric pressure in the chamber was attained by a rotary pump and regulated only by watching any change.

Heart beats were recorded in the case of high pressure experiment with a lever attached to the heart apex on the small kymograph, the movement of which was started or stopped from outside of the box by an electrical switch at will.

Perfusion fluid was composed as follows :

$\begin{array}{llll}\mathrm{NaCl} & 0.65 \% & \mathrm{Ha}_{2} \mathrm{HPO}_{4} & 0.0069 \% \\ \mathrm{KCl} & 0.02 \% & \mathrm{NaH}_{2} \mathrm{PO}_{4} & 0.016 \%\end{array}$




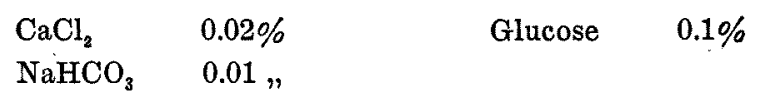

The $\mathrm{pH}$ was potentiometrically 7.3 .

Experimental procedures : About 20-30 minutes after having finished the perfusion preparation and arrangements for the pressure experiment conductivity of the perfusion fluid was measured at the normal atmospheric pressure, and then the pressure in the experimental chamber was changed by the above mentioned apparatus. In the high pressure experiment the pressure was increased from zero pound (the atmospheric pressure) through $10,20,30$ to 40 pounds, taking about 40 minutes to attain the final pressure. At each pressure of the first three kinds, it was kept at the same for 5 minutes and the conductivity was measured and at the same time the kymograph was started to record the heart beats of frog for a few minutes. The 40 pound pressure was kept for 2 hours, during which the conductivity was measured every 30 minutes and the heart beats were recorded. Pressure recovery was performed in just the reverse order and the conductivity measurement and recording of the heart beats were again taken.

Low pressure experiment was done in the same manner as the high pressure. The pressure experimented with in the chamber was $400 \mathrm{~mm}$ $\mathrm{Hg}$. lower than the atmospheric pressure at that time.

\section{EXPERIMENT.}

1. Conductivity of Ringer's solution under high atmospheric pressure.

No change of conductivity was found in Ringer's solution which was to be used as the perfusion fluid, under influence of the high pressure effected in the manner above described.

2. Conductivity of perfusion fluid of frog heart under the normal atmospheric pressure (Table 1 and Figure).

Conductivity of perfusion fluid of frog heart was measured during three hours. Slight changes were noticed during the three hours, especially in the first thirty minutes, but the direction of change seemed not to be constant. 
TAble 1.

Conductivity of perfusion fuid of frog heart under the normal pressure.

\begin{tabular}{|c|c|c|c|c|c|c|c|c|}
\hline \multirow{2}{*}{ No. } & \multicolumn{8}{|c|}{$\begin{array}{l}\text { Time (min.) after beginning of experiment, specific conductivity } \times 10^{-5} \\
\text { and ratio of the value at various period to that at the beginning }\end{array}$} \\
\hline & 0 & 20 & 40 & 60 & 80 & 100 & 120 & 140 \\
\hline \multirow{2}{*}{1} & 1087 & 1083 & 1083 & 1080 & 1075 & 1075 & 1080 & 1080 \\
\hline & 1.00 & 1.00 & 1.00 & 0.99 & 1.00 & 0.99 & 0.99 & 0.99 \\
\hline \multirow{2}{*}{2} & 1106 & 1106 & 1115 & 1117 & 1113 & 1113 & 1115 & 1124 \\
\hline & 1.00 & 1.00 & 1.01 & 1.01 & 1.01 & 1.01 & 1.01 & 1.02 \\
\hline \multirow{2}{*}{3} & 1071 & 1068 & 1078 & 1087 & 1087 & 1078 & 1078 & 1078 \\
\hline & 1.00 & 1.00 & 1.01 & 1.01 & 1.01 & 1.01 & 1.01 & 1.01 \\
\hline \multirow[b]{2}{*}{4} & 996 & 978 & 978 & 989 & 989 & 989 & 989 & 989 \\
\hline & 1.00 & 0.98 & 0.98 & 0.99 & 0.99 & 0.99 & 0.99 & 0.99 \\
\hline \multirow{2}{*}{5} & 1004 & 1009 & 999 & 988 & 988 & 988 & 1002 & 1002 \\
\hline & 1.00 & 1.00 & 0.99 & 0.98 & 0.98 & 0.98 & 1.00 & 1.00 \\
\hline \multirow[b]{2}{*}{6} & 820 & 806 & 806 & 812 & 812 & 812 & 812 & 812 \\
\hline & 1.00 & 0.98 & 0.98 & 0.99 & 0.99 & 0.99 & 0.99 & 0.99 \\
\hline \multirow{2}{*}{7} & 967 & 963 & 961 & 962 & 962 & 962 & 961 & 963 \\
\hline & 1.00 & 1.00 & 0.99 & 0.99 & 0.99 & 0.99 & 0.99 & 1.00 \\
\hline \multirow{2}{*}{8} & 843 & 888 & 888 & 903 & $\dot{9} 19$ & 920 & 920 & 920 \\
\hline & 1.00 & 1.05 & 1.05 & 1.07 & 1.09 & 1.09 & 1.09 & 1.09 \\
\hline \multirow{2}{*}{9} & 967 & 966 & 966 & 966 & 966 & 966 & 966 & 966 \\
\hline & 1.00 & 1.00 & 1.00 & 1.00 & 1.00 & 1.00 & 1.00 & 1.00 \\
\hline \multirow{2}{*}{10} & 932 & 915 & 918 & 918 & 918 & 925 & 935 & 935 \\
\hline & 1.00 & 0.98 & 0.98 & 0.98 & 0.98 & 0.99 & 1.00 & 1.00 \\
\hline Mean & $\begin{array}{r}978 \\
1.00\end{array}$ & $\begin{array}{r}978 \\
1.00 \\
\pm 0.004\end{array}$ & $\begin{array}{r}979 \\
1.00 \\
\pm 0.005\end{array}$ & $\begin{array}{r}983 \\
1.00 \\
\pm 0.006\end{array}$ & $\begin{array}{r}983 \\
1.00 \\
\pm 0.007\end{array}$ & $\begin{array}{r}983 \\
1.00 \\
\pm 0.007\end{array}$ & $\begin{array}{r}986 \\
1.01 \\
\pm 0.006\end{array}$ & $\begin{array}{r}987 \\
1.01 \\
\pm \mathbf{0 . 0 0 6}\end{array}$ \\
\hline
\end{tabular}

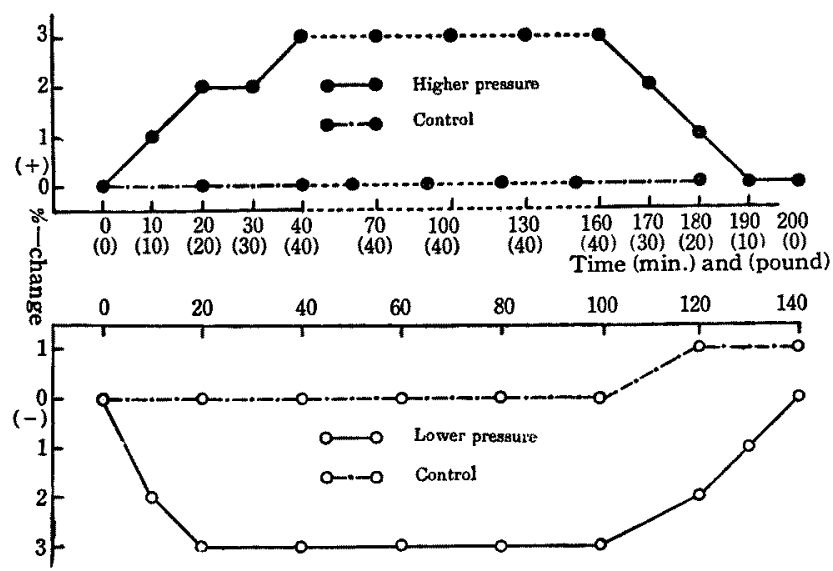

Fig. Percentage (\%) change of conductivity of perfusion fluid of frog heart under abnormal atmospheric pressure. 
3. Conductivity of perfusion fluid of frog heart under high atmospheric pressure (Table 2 and Figure).

Table 2.

Conductivity $\left(\times 10^{-5}\right)$ of perfusion fluid of frog heart under high atmospheric pressure (10-40 pounds).

\begin{tabular}{|c|c|c|c|c|c|c|c|c|c|c|c|c|c|}
\hline No. $(\mathrm{P})$ & $\begin{array}{c}0 \\
(0)\end{array}$ & $\begin{array}{c}10 \\
(10)\end{array}$ & $\begin{array}{c}20 \\
(20)\end{array}$ & $\begin{array}{c}30 \\
(\mathbf{3 0})\end{array}$ & $\begin{array}{c}40 \\
(40)\end{array}$ & $\begin{array}{c}70 \\
(40)\end{array}$ & $\begin{array}{c}100 \\
(40)\end{array}$ & $\begin{array}{l}130 \\
(40)\end{array}$ & $\begin{array}{c}160 \\
(40)\end{array}$ & $\begin{array}{r}170 \\
(30)\end{array}$ & $\begin{array}{c}180 \\
(20)\end{array}$ & $\begin{array}{r}190 \\
(10)\end{array}$ & $\begin{array}{l}200 \\
(0)\end{array}$ \\
\hline \multirow{2}{*}{1} & 1782 & 1802 & 1802 & 1823 & 1836 & 1836 & 1832 & 1832 & 1832 & 1823 & 1802 & 1802 & 1782 \\
\hline & 1.00 & 1.01 & 1.01 & 1.02 & 1.03 & 1.03 & 1.03 & 1.03 & 1.03 & 1.02 & 1.01 & 1.01 & 1.00 \\
\hline \multirow[t]{2}{*}{2} & 1782 & 1802 & 1823 & 1823 & 1867 & 1867 & 1867 & 1867 & 1823 & 1823 & 1741 & 1741 & 1741 \\
\hline & 1.00 & 1.01 & 1.02 & 1.02 & 1.05 & 1.05 & 1.05 & 1.05 & 1.02 & 1.02 & 0.98 & 0.98 & 0.98 \\
\hline \multirow{2}{*}{3} & 1782 & 1782 & 1802 & 1808 & 1814 & 1823 & 1841 & 1841 & 1841 & 1867 & 1867 & 1867 & 1867 \\
\hline & 1.00 & 1.00 & 1.01 & 1.02 & 1.02 & 1.02 & 1.03 & 1.03 & 1.03 & 1.05 & 1.05 & 1.05 & 1.05 \\
\hline \multirow{2}{*}{4} & 1782 & 1802 & 1802 & 1823 & 1832 & 1832 & 1832 & 1832 & 1823 & 1823 & 1823 & 1823 & 1802 \\
\hline & 1.00 & 1.01 & 1.01 & 1.02 & 1.03 & 1.03 & 1.03 & 1.03 & 1.02 & 1.02 & 1.02 & 1.02 & 1.01 \\
\hline \multirow[t]{2}{*}{$\mathbf{5}$} & 1782 & & 1802 & 1823 & 1836 & 1836 & 1836 & 1836 & 1836 & 1823 & 1802 & & 1782 \\
\hline & 1.00 & & 1.01 & 1.02 & 1.03 & 1.03 & 1.03 & 1.03 & 1.03 & 1.02 & 1.01 & & 1.00 \\
\hline \multirow{2}{*}{6} & 1782 & 1802 & 1823 & 1836 & 1836 & 1836 & 1836 & 1836 & 1836 & 1823 & 1802 & 1802 & 1802 \\
\hline & 1.00 & 1.01 & 1.02 & 1.03 & 1.03 & 1.03 & 1.03 & 1.03 & 1.03 & 1.02 & 1.01 & 1.01 & 1.01 \\
\hline \multirow{2}{*}{7} & 1782 & & 1802 & 1823 & 1823 & 1823 & 1823 & 1823 & 1823 & 1802 & 1802 & 1802 & 1782 \\
\hline & 1.00 & & 1.01 & 1.02 & 1.02 & 1.02 & 1.02 & 1.02 & 1.02 & 1.01 & 1.01 & 1.01 & 1.00 \\
\hline \multirow{2}{*}{8} & 1782 & 1782 & 1811 & 1815 & 1867 & 1836 & 1836 & 1836 & 1836 & 1823 & 1823 & 1782 & 1782 \\
\hline & 1.00 & 1.00 & 1.02 & 1.02 & 1.05 & 1.03 & 1.03 & 1.03 & 1.03 & 1.02 & 1.02 & 1.00 & 1.00 \\
\hline \multirow{2}{*}{9} & 1782 & 1782 & 1802 & 1823 & 1836 & 1836 & 1836 & 1836 & 1836 & 1823 & 1802 & 1802 & 1782 \\
\hline & 1.00 & 1.00 & 1.01 & 1.02 & 1.03 & 1.03 & 1.03 & 1.03 & 1.03 & 1.02 & 1.01 & 1.01 & 1.00 \\
\hline \multirow{2}{*}{10} & 1782 & 1802 & 1823 & 1828 & 1836 & 1836 & 1836 & 1836 & 1836 & 1802 & 1802 & 1741 & 1741 \\
\hline & 1.00 & 1.01 & 1.02 & 1.03 & 1.03 & 1.03 & 1.03 & 1.03 & 1.03 & 1.01 & 1.01 & 0.98 & 0.98 \\
\hline \multirow{2}{*}{11} & 1782 & 1802 & 1807 & 1823 & 1823 & 1823 & 1836 & 1836 & 1836 & 1823 & 1802 & 1802 & 1782 \\
\hline & 1.00 & 1.01 & 1.01 & 1.02 & 1.02 & 1.02 & 1.03 & 1.03 & 1.03 & 1.02 & 1.01 & 1.01 & 1.00 \\
\hline \multirow{2}{*}{12} & 1782 & 1802 & 1823 & 1823 & 1836 & 1836 & 1836 & 1836 & 1836 & 1823 & 1823 & 1802 & 1782 \\
\hline & 1.00 & 1.01 & 1.02 & 1.02 & 1.03 & 1.03 & 1.03 & 1.03 & 1.03 & 1.02 & 1.02 & 1.01 & 1.00 \\
\hline \multirow[t]{2}{*}{13} & 1782 & 1815 & 1832 & 1836 & 1836 & 1836 & 1836 & 1836 & 1836 & 1823 & 1815 & 1802 & 1782 \\
\hline & 1.00 & 1.02 & 1.03 & 1.03 & 1.03 & 1.03 & 1.03 & 1.03 & 1.03 & 1.02 & 1.02 & 1.01 & 1.00 \\
\hline \multirow[t]{2}{*}{14} & 1782 & 1802 & 1823 & 1823 & 1823 & 1823 & 1823 & 1823 & 1823 & 1823 & 1802 & 1761 & 1741 \\
\hline & 1.00 & 1.01 & 1.02 & 1.02 & 1.02 & 1.02 & 1.02 & 1.02 & 1.02 & 1.02 & 1.01 & 0.99 & 0.98 \\
\hline \multirow[t]{2}{*}{15} & 1782 & 1808 & 1802 & 1823 & 1836 & 1836 & 1836 & 1836 & 1836 & 1823 & 1823 & 1802 & 1802 \\
\hline & 1.00 & 1.02 & 1.01 & 1.02 & 1.03 & 1.03 & 1.03 & 1.03 & 1.03 & 1.02 & 1.02 & 1.01 & 1.01 \\
\hline \multirow[t]{2}{*}{16} & 1782 & 1802 & 1815 & 1823 & 1823 & 1823 & 1823 & 1823 & 1802 & 1802 & 1782 & 1761 & 1753 \\
\hline & 1.00 & 1.01 & 1.02 & 1.02 & 1.02 & 1.02 & 1.02 & 1.02 & 1.01 & 1.01 & 1.00 & 0.99 & 0.98 \\
\hline \multirow{2}{*}{17} & 1782 & & & & 1867 & 1867 & 1867 & 1867 & 1867 & 1823 & 1823 & 1802 & 1782 \\
\hline & 1.00 & & & & 1.05 & 1.05 & 1.05 & 1.05 & 1.05 & 1.02 & 1.02 & 1.01 & 1.00 \\
\hline \multirow[t]{2}{*}{18} & 1782 & & & & 1802 & 1823 & 1823 & 1823 & 1823 & 1808 & 1802 & 1802 & 1782 \\
\hline & 1.00 & & & & 1.01 & 1.02 & 1.02 & 1.02 & 1.02 & 1.02 & 1.01 & 1.01 & 1.00 \\
\hline \multirow[t]{2}{*}{19} & 1782 & & 1802 & 1823 & 1802 & 1802 & 1802 & 1802 & 1761 & 1741 & 1721 & 1702 & 1702 \\
\hline & 1.00 & & 1.01 & 1.02 & 1.01 & 1.01 & 1.01 & 1.01 & 0.99 & 0.98 & 0.97 & 0.95 & 0.95 \\
\hline \multirow[t]{2}{*}{20} & 1782 & 1802 & 1802 & 1802 & 1802 & 1802 & 1823 & 1823 & 1823 & 1823 & 1808 & 1808 & 1802 \\
\hline & 00 & 1.01 & 1.01 & 1.01 & 1.01 & 1.01 & 1.02 & 1.02 & 1.02 & 1.02 & 1.02 & 1.02 & 1.01 \\
\hline & 17 & 1799 & 1811 & 1822 & 1832 & 1832 & 1834 & 1834 & 1833 & 1822 & 1803 & 1789 & 1789 \\
\hline Mean & & 01 & 1.02 & 1.02 & 1.03 & 1.03 & 1.03 & 1.03 & 1.03 & 1.02 & 1.01 & 1.00 & 1.00 \\
\hline & 1 & & 1 & .001 & \pm .002 & $\begin{array}{c} \pm .002 \\
0.002\end{array}$ & \pm & $\stackrel{ \pm}{0.001}$ & \pm .002 & \pm .002 & $\mid \begin{array}{l} \pm \\
0.002\end{array}$ & $\stackrel{ \pm}{0.003}$ & $\mid \pm .002$ \\
\hline
\end{tabular}

Min : time after beginning of pressureincease.

$(\mathrm{P})$ : pounds of added pressure. 
Distinct increase of the conductivity was always found according to the pressure increase, the conductivity during stay at 40 pounds being kept almost at the same level.

These results show that there is some ion exchange between perfusion fluid and the heart tissue, resulting ion increase in the former. This process seems to occur very quickly following change of the pressure.

4. Conductivity of perfusion fluid of frog heart in low atmospheric pressure (Tables 3, 4 and Figure).

The conductivity changed according to the pressure variation just in the reverse direction. That is the conductivity decreased following

TABLE 3.

Conductivity of perfusion fluid of frog heart under the normal atmospheric pressure.

\begin{tabular}{|c|c|c|c|c|c|c|c|}
\hline \multirow{2}{*}{ No. } & \multicolumn{7}{|c|}{$\begin{array}{l}\text { Time (min.) after beginning of the experiment, specific conductivity } \times 10^{-5} \\
\text { and ratio of the value at various period to that at the beginning. }\end{array}$} \\
\hline & 0 & 30 & 60 & 90 & 120 & 150 & 180 \\
\hline 1 & $\begin{array}{r}1782 \\
1.00\end{array}$ & $\begin{array}{r}1777 \\
1.00\end{array}$ & $\begin{array}{r}1782 \\
1.00\end{array}$ & $\begin{array}{r}1782 \\
1.00\end{array}$ & $\begin{array}{r}1777 \\
1.00\end{array}$ & $\begin{array}{r}1782 \\
1.00\end{array}$ & $\begin{array}{r}1782 \\
1.00\end{array}$ \\
\hline $\mathbf{8}$ & $\begin{array}{l}1782 \\
1.00\end{array}$ & $\begin{array}{r}1782 \\
1.00\end{array}$ & $\begin{array}{r}1782 \\
1.00\end{array}$ & $\begin{array}{r}1782 \\
1.00\end{array}$ & $\begin{array}{r}1782 \\
1.00\end{array}$ & $\begin{array}{r}1782 \\
1.00\end{array}$ & $\begin{array}{r}1782 \\
1.00\end{array}$ \\
\hline 3 & $\begin{array}{r}1782 \\
1.00\end{array}$ & $\begin{array}{r}1782 \\
1.00\end{array}$ & $\begin{array}{r}1782 \\
1.00\end{array}$ & $\begin{array}{r}1797 \\
1.01\end{array}$ & $\begin{array}{l}1777 \\
1.00\end{array}$ & $\begin{array}{r}1777 \\
1.00\end{array}$ & $\begin{array}{r}1782 \\
1.00\end{array}$ \\
\hline 4 & $\begin{array}{r}1782 \\
1.00\end{array}$ & $\begin{array}{r}1782 \\
1.00\end{array}$ & $\begin{array}{r}1782 \\
1.00\end{array}$ & $\begin{array}{r}1782 \\
1.00\end{array}$ & $\begin{array}{r}1782 \\
1.00\end{array}$ & $\begin{array}{c}1782, \\
1.00^{\prime}\end{array}$ & $\begin{array}{r}1782 \\
1.00\end{array}$ \\
\hline 5 & $\begin{array}{r}1782 \\
1.00\end{array}$ & $\begin{array}{r}1782 \\
1.00\end{array}$ & $\begin{array}{l}1782 \\
1.00\end{array}$ & $\begin{array}{r}1782 \\
1.00\end{array}$ & $\begin{array}{r}1782 \\
1.00\end{array}$ & $\begin{array}{r}1782 \\
1.00\end{array}$ & $\begin{array}{r}1798 \\
1.01\end{array}$ \\
\hline 6 & $\begin{array}{r}1782 \\
1.00\end{array}$ & $\begin{array}{r}1782 \\
1.00\end{array}$ & $\begin{array}{r}1782 \\
1.00\end{array}$ & $\begin{array}{r}1782 \\
1.00\end{array}$ & $\begin{array}{r}1782 \\
1.00\end{array}$ & $\begin{array}{r}1782 \\
1.00\end{array}$ & $\begin{array}{r}1782 \\
1.00\end{array}$ \\
\hline 7 & $\begin{array}{r}1782 \\
1.00\end{array}$ & $\begin{array}{r}1782 \\
1.00\end{array}$ & $\begin{array}{r}1782 \\
1.00\end{array}$ & $\begin{array}{r}1777 \\
1.00\end{array}$ & $\begin{array}{r}1777 \\
1.00\end{array}$ & $\begin{array}{r}1782 \\
1.00\end{array}$ & $\begin{array}{r}1782 \\
1.00\end{array}$ \\
\hline 8 & $\begin{array}{r}1782 \\
1.00\end{array}$ & $\begin{array}{r}1782 \\
1.00\end{array}$ & $\begin{array}{r}1782 \\
1.00\end{array}$ & $\begin{array}{r}1782 \\
1.00\end{array}$ & $\begin{array}{r}1782 \\
1.00\end{array}$ & $\begin{array}{r}1782 \\
1.00\end{array}$ & $\begin{array}{r}1782 \\
1.00\end{array}$ \\
\hline 9 & $\begin{array}{r}1782 \\
1.00\end{array}$ & $\begin{array}{r}1741 \\
0.98\end{array}$ & $\begin{array}{r}1786 \\
1.00\end{array}$ & $\begin{array}{r}1782 \\
1.00\end{array}$ & $\begin{array}{r}1782 \\
1.00\end{array}$ & $\begin{array}{r}1782 \\
1.00\end{array}$ & $\begin{array}{r}1782 \\
1.00\end{array}$ \\
\hline 10 & $\begin{array}{r}1782 \\
1.00\end{array}$ & $\begin{array}{r}1765 \\
0.99\end{array}$ & $\begin{array}{r}1782 \\
1.00\end{array}$ & $\begin{array}{r}1782 \\
1.00\end{array}$ & $\begin{array}{r}1782 \\
1.00\end{array}$ & $\begin{array}{r}1782 \\
1.00\end{array}$ & $\begin{array}{r}1782 \\
1.00\end{array}$ \\
\hline 11 & $\begin{array}{l}1782 \\
1.00\end{array}$ & $\begin{array}{r}1782 \\
1.00\end{array}$ & $\begin{array}{r}1782 \\
1.00\end{array}$ & $\begin{array}{r}1782 \\
1.00\end{array}$ & $\begin{array}{r}1782 \\
1.00\end{array}$ & $\begin{array}{r}1782 \\
1.00\end{array}$ & $\begin{array}{r}1782 \\
1.00\end{array}$ \\
\hline Mean & $\begin{array}{l}1782 \\
1.00\end{array}$ & $\begin{array}{r}1776 \\
1.00 \\
\pm 0.001\end{array}$ & $\begin{array}{r}1782 \\
1.00 \\
\pm 0.000\end{array}$ & $\begin{array}{r}1783 \\
1.00 \\
\pm 0.001\end{array}$ & $\begin{array}{r}1781 \\
1.00 \\
\pm 0.000\end{array}$ & $\begin{array}{r}1782 \\
1.00 \\
\pm 0.000\end{array}$ & $\begin{array}{r}1783 \\
1.00 \\
\pm 0.001\end{array}$ \\
\hline
\end{tabular}




\section{TABLE 4.}

Conductivity of perfusion fluid of frog heart under low atmospheric pressure $(-400 \mathrm{~mm} \mathrm{Hg}$.)

\begin{tabular}{|c|c|c|c|c|c|c|c|c|c|c|}
\hline \multirow{2}{*}{ No. } & \multicolumn{10}{|c|}{$\begin{array}{l}\text { Time (min.) after beginning of pressure decrease, specific conductivity } \times 10^{-6} \\
\text { and ratio of the value under low pressure to that before pressure change. }\end{array}$} \\
\hline & before & immed. & 20 & 40. & 60 & 80 & 100 & 120 & $\begin{array}{c}\text { immed. } \\
\text { after }\end{array}$ & 20 \\
\hline 1 & $\begin{array}{r}1104 \\
1.00\end{array}$ & $\begin{array}{r}1082 \\
0.98\end{array}$ & $\begin{array}{r}1075 \\
0.97\end{array}$ & $\begin{array}{r}1075 \\
0.97\end{array}$ & $\begin{array}{r}1077 \\
0.98\end{array}$ & $\begin{array}{r}1077 \\
0.98\end{array}$ & $\begin{array}{r}1079 \\
0.98\end{array}$ & $\begin{array}{r}1079 \\
0.98\end{array}$ & $\begin{array}{r}1104 \\
1.00\end{array}$ & $\begin{array}{r}1104 \\
1.00\end{array}$ \\
\hline 2 & $\begin{array}{r}1092 \\
1.00\end{array}$ & $\begin{array}{r}1092 \\
1.00\end{array}$ & $\begin{array}{r}1097 \\
1.00\end{array}$ & $\begin{array}{r}1099 \\
1.01\end{array}$ & $\begin{array}{r}1095 \\
1.00\end{array}$ & $\begin{array}{r}1095 \\
1.00\end{array}$ & $\begin{array}{r}1095 \\
1.00\end{array}$ & $\begin{array}{r}1103 \\
1.01\end{array}$ & $\begin{array}{r}1108 \\
1.01\end{array}$ & $\begin{array}{r}1121 \\
1.03\end{array}$ \\
\hline 3 & $\begin{array}{r}1105 \\
1.00\end{array}$ & $\begin{array}{r}1093 \\
0.99\end{array}$ & $\begin{array}{r}1085 \\
0.98\end{array}$ & $\begin{array}{r}1086 \\
0.98\end{array}$ & $\begin{array}{r}1089 \\
0.99\end{array}$ & $\begin{array}{r}1095 \\
0.99\end{array}$ & $\begin{array}{r}1095 \\
0.99\end{array}$ & $\begin{array}{r}1095 \\
0.99\end{array}$ & $\begin{array}{r}1105 \\
1.00\end{array}$ & $\begin{array}{r}1105 \\
1.00\end{array}$ \\
\hline 4 & $\begin{array}{r}1020 \\
1.00\end{array}$ & $\begin{array}{r}1000 \\
0.98\end{array}$ & $\begin{array}{r}993 \\
0.97\end{array}$ & $\begin{array}{r}993 \\
0.97\end{array}$ & $\begin{array}{r}993 \\
0.97\end{array}$ & $\begin{array}{r}993 \\
0.97\end{array}$ & $\begin{array}{r}993 \\
0.97\end{array}$ & $\begin{array}{r}993 \\
0.97\end{array}$ & $\begin{array}{r}992 \\
0.97\end{array}$ & $\begin{array}{r}992 \\
0.97\end{array}$ \\
\hline 5 & $\begin{array}{r}1000 \\
1.00\end{array}$ & $\begin{array}{r}993 \\
0.99\end{array}$ & $\begin{array}{r}984 \\
0.98\end{array}$ & $\begin{array}{r}976 \\
0.98\end{array}$ & $\begin{array}{r}976 \\
0.98\end{array}$ & $\begin{array}{r}975 \\
0.98\end{array}$ & $\begin{array}{r}975 \\
0.98\end{array}$ & $\begin{array}{r}979 \\
0.98\end{array}$ & $\begin{array}{r}1001 \\
1.00\end{array}$ & $\begin{array}{r}1001 \\
1.00\end{array}$ \\
\hline 6 & $\begin{array}{r}833 \\
1.00\end{array}$ & $\begin{array}{r}818 \\
0.93\end{array}$ & $\begin{array}{r}799 \\
0.90\end{array}$ & $\begin{array}{r}796 \\
0.90\end{array}$ & $\begin{array}{r}793 \\
0.90\end{array}$ & $\begin{array}{r}793 \\
0.90\end{array}$ & $\begin{array}{r}793 \\
0.90\end{array}$ & $\begin{array}{r}793 \\
0,90\end{array}$ & $\begin{array}{r}813 \\
0.92\end{array}$ & $\begin{array}{r}830 \\
0.94\end{array}$ \\
\hline 7 & $\begin{array}{r}971 \\
1.00\end{array}$ & $\begin{array}{r}957 \\
0.99\end{array}$ & $\begin{array}{r}949 \\
0.98\end{array}$ & $\begin{array}{r}949 \\
0.98\end{array}$ & $\begin{array}{r}949 \\
0.98\end{array}$ & $\begin{array}{r}949 \\
0.98\end{array}$ & $\begin{array}{r}949 \\
0.98\end{array}$ & $\begin{array}{r}950 \\
0.98\end{array}$ & $\begin{array}{r}964 \\
0.99\end{array}$ & $\begin{array}{r}971 \\
1.00\end{array}$ \\
\hline 8 & $\begin{array}{r}827 \\
1.00\end{array}$ & $\begin{array}{r}810 \\
0.98\end{array}$ & $\begin{array}{r}799 \\
0.97\end{array}$ & $\begin{array}{r}799 \\
0.97\end{array}$ & $\begin{array}{r}800 \\
0.97\end{array}$ & $\begin{array}{r}826 \\
1.00\end{array}$ & $\begin{array}{r}840 \\
1.02\end{array}$ & $\begin{array}{r}852 \\
1.03\end{array}$ & $\begin{array}{r}859 \\
1.04\end{array}$ & $\begin{array}{r}875 \\
1.06\end{array}$ \\
\hline 9 & $\begin{array}{r}979 \\
1.00\end{array}$ & $\begin{array}{r}965 \\
0.99\end{array}$ & $\begin{array}{r}958 \\
0.98\end{array}$ & $\begin{array}{r}952 \\
0.97\end{array}$ & $\begin{array}{r}952 \\
0.97\end{array}$ & $\begin{array}{r}952 \\
0.97\end{array}$ & $\begin{array}{r}951 \\
0.97\end{array}$ & $\begin{array}{r}951 \\
0.97\end{array}$ & $\begin{array}{r}956 \\
0.98\end{array}$ & $\begin{array}{r}971 \\
0.99\end{array}$ \\
\hline 10 & $\begin{array}{r}941 \\
1.00\end{array}$ & $\begin{array}{r}907 \\
0.96\end{array}$ & $\begin{array}{r}882 \\
0.94\end{array}$ & $\begin{array}{r}882 \\
0.94\end{array}$ & $\begin{array}{r}882 \\
0.94\end{array}$ & $\begin{array}{r}882 \\
0.94\end{array}$ & $\begin{array}{r}881 \\
0.94\end{array}$ & $\begin{array}{r}888 \\
0.94\end{array}$ & $\begin{array}{r}913 \\
0.97\end{array}$ & $\begin{array}{r}913 \\
0.97\end{array}$ \\
\hline Mean & $\begin{array}{r}982 \\
1.00\end{array}$ & $\begin{array}{r}972 \\
0.98 \\
\pm 0.004\end{array}$ & $\begin{array}{r}962 \\
0.97 \\
\pm 0.006\end{array}$ & $\begin{array}{r}961 \\
0.97 \\
+0.006\end{array}$ & $\begin{array}{r}961 \\
0.97 \\
+0.006\end{array}$ & $\begin{array}{r}965 \\
0.97 \\
+0.007\end{array}$ & $\begin{array}{r}965 \\
0.97 \\
\pm 0.007\end{array}$ & $\begin{array}{r}968 \\
0.98 \\
+0.008\end{array}$ & $\begin{array}{r}982 \\
0.99 \\
\pm 0.007\end{array}$ & $\begin{array}{r}9.88 \\
1.00 \\
+0.007\end{array}$ \\
\hline
\end{tabular}

lowering of the pressure and recovered according to the pressure recovery. Though the conductivity of the control (Table 3) showed an increase at the end of 2 hours, the decrease during the lower pressure was beyond doubt. Here again we found some ion exchange between fluid and heart tissue, resulting in ion decrease in the former.

\section{On the heart beats of perfusion frog heart under abnormal atmospheric pressure (Table 5).}

As can be seen from the table height of heart beat showed only slight change during three hour observation under high atmospheric 
Table 5a.

Height (Ht, em in kymographic record) and frequency ( $F r q$, in 8 seconds) of heart beat of frog under high atmospheric pressure.

\begin{tabular}{|c|c|c|c|c|c|c|c|c|c|c|c|c|c|c|}
\hline \multirow{2}{*}{ No. } & Min. & 0 & 10 & 20 & 30 & 40 & 70 & 100 & 130 & 160 & 170 & 180 & 190 & 200 \\
\hline & Pou & $(0)$ & $(10)$ & $(20)$ & (30) & $(40)$ & $(40)$ & $(40)$ & $(40)$ & $(40)$ & $(30)$ & $(20)$ & $(10)$ & (0) \\
\hline \multirow{2}{*}{1} & & .70 & 1.80 & 97 & 1.87 & 1.91 & .96 & 1.86 & 1.70 & 1.55 & 1.28 & 1.15 & 1.00 & 0.79 \\
\hline & & 11 & 10 & 10 & 10 & 11 & 12 & 12 & 11 & 10 & 10 & 10 & 11 & 11 \\
\hline \multirow{2}{*}{2} & & 1.30 & 1.40 & 1.60 & 1.80 & 1.90 & 1.87 & 1.90 & 1.90 & 1.87 & 1.70 & 1.50 & 1.45 & 1.43 \\
\hline & & 12 & 12 & 13 & 13 & 13 & 13 & 13 & 12 & 12 & 12 & 10 & 9 & \\
\hline \multirow{2}{*}{3} & & 1.57 & 1.48 & 1.45 & 1.47 & 1.40 & 1.40 & 1.39 & 1.38 & .48 & 58 & 1.60 & 60 & .60 \\
\hline & & 9 & 14 & 13 & 13 & 13 & 13 & 13 & 13 & 8 & 9 & 8 & 8 & \\
\hline \multirow[t]{2}{*}{4} & & 1.81 & 1.79 & 1.50 & 1.45 & 1.38 & 1.12 & 1.18 & 1.22 & .27 & 1.31 & 1.31 & .25 & 1.20 \\
\hline & & & 12 & 3 & 13 & 15 & 4 & 13 & 13 & 13 & 13 & 13 & 14 & 14 \\
\hline \multirow[t]{2}{*}{5} & & 1.30 & 1.11 & 1.10 & 1.07 & 1.03 & 0.94 & 0.92 & 0.90 & 0.88 & .83 & 0.84 & .90 & 0.90 \\
\hline & & 7 & 11 & 12 & 12 & 11 & 11 & 11 & 6 & 6 & 6 & 5 & 6 & \\
\hline \multirow{2}{*}{6} & & 1.55 & 1.52 & 1.55 & & 1.58 & 1.46 & 1.35 & 1.19 & 1.08 & 0.99 & 0.90 & & \\
\hline & & 1 & 10 & 0 & & 11 & 1 & 10 & 11 & 13 & 6 & 6 & & \\
\hline \multirow[t]{2}{*}{7} & & 1.46 & 1.75 & 1.65 & 1.56 & 1.56 & 1.65 & 1.50 & 1.45 & 1.42 & .36 & 1.30 & 1.19 & \\
\hline & & A & 13 & 11 & 12 & 13 & 12 & 12 & 11 & 10 & 9 & 10 & 10 & \\
\hline \multirow[t]{2}{*}{8} & & 1.55 & 1.51 & 1.67 & 1.69 & 1.61 & 1.35 & 0.97 & 0.94 & 0.95 & 0.81 & 0.81 & 0.78 & 0.75 \\
\hline & & 2 & 11 & 1 & 12 & 11 & 10 & 11 & 11 & 10 & 12 & 13 & 12 & 12 \\
\hline 9 & $I$ & 1.95 & 2.28 & 2.47 & 2.55 & 2.58 & 2.35 & 2.34 & 2.18 & 2.10 & .08 & 1.90 & 1.90 & 1.70 \\
\hline & & 13 & 11 & 13 & 13 & 12 & 12 & 1 & 12 & 12 & 8 & 8 & 8 & \\
\hline 1 & & 1.51 & & 1.65 & 1.56 & 1.64 & 1.80 & 1.90 & 1.98 & 1.20 & 95 & 1.87 & .81 & 1.80 \\
\hline \multirow[b]{2}{*}{11} & & & & & 50 & 11 & 11 & 65 & 11 & 1 & 0 & 10 & 10 & 11 \\
\hline & & $\begin{array}{r}1.00 \\
13\end{array}$ & $\begin{array}{r}1.05 \\
14\end{array}$ & $\begin{array}{r}1.40 \\
13\end{array}$ & 13 & $\begin{array}{r}1.00 \\
13\end{array}$ & 13 & 13 & 13 & 13 & $\begin{array}{r}+7 \\
13\end{array}$ & 13 & 12 & $\begin{array}{r}+40 \\
12\end{array}$ \\
\hline \multirow{2}{*}{1} & $\mathbf{H}$ & 0.81 & & 0.81 & 1.04 & 1.07 & 1.07 & $1 \cdot 10$ & 1.10 & 1.05 & 0.83 & 0.75 & & 0.90 \\
\hline & & 14 & & 13 & 12 & 12 & 13 & 10 & 10 & 8 & 8 & 7 & & \\
\hline \multirow{2}{*}{ Mean } & & 1.51 & 1.62 & 1.58 & 1.60 & 1.60 & 1.54 & 1.5 & 1.46 & 1.37 & 1.35 & 1.28 & 1.32 & 1.25 \\
\hline & & : & 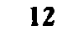 & 12 & 17 & 1 & 12 & 12 & 11 & 11 & 10 & & 10 & \\
\hline
\end{tabular}

TABle $5 \mathrm{~b}$.

Height (Ht, em in kymographic record) and frequency (Frq, in 8 seconds) of heart beat of frog under the normal atmospheric pressure.

\begin{tabular}{|c|c|c|c|c|c|c|c|c|c|c|c|c|c|c|}
\hline \multirow{3}{*}{ No. } & \multicolumn{14}{|c|}{ Time (min.) after beginning of observation } \\
\hline & \multicolumn{2}{|c|}{$\mathbf{0}$} & \multicolumn{2}{|c|}{30} & \multicolumn{2}{|c|}{60} & \multicolumn{2}{|c|}{90} & \multicolumn{2}{|c|}{120} & \multicolumn{2}{|c|}{150} & \multicolumn{2}{|c|}{180} \\
\hline & Ht & Frq & Hit & Frq & Ht & Frq & $\mathrm{Ht}$ & Frq & Ht & Frq & $\mathrm{Ht}$ & Frq & Ht & Frq \\
\hline 1 & 2.10 & 10 & 1.90 & 9 & 1.85 & 6 & 2.05 & 6 & 1.93 & 6 & 1.80 & 6 & 1.80 & 6 \\
\hline 2 & 1.55 & 14 & 1.50 & 11 & 1.50 & 11 & 1.75 & 9 & 1.80 & 8 & 1.50 & 7 & 1.47 & 7 \\
\hline 3 & 1.90 & 11 & 1.90 & 11 & 1.85 & 11 & 2.00 & 11 & 1.95 & 12 & 2.00 & 11 & 1.80 & 10 \\
\hline 4 & 1.30 & 12 & 1.20 & 12 & 1.05 & 13 & 1.00 & 12 & 0.95 & 12 & 0.95 & 13 & 0.87 & 12 \\
\hline 5 & 1.40 & 11 & 1.55 & 6 & 1.45 & 6 & 1.35 & 6 & 1.40 & 6 & 1.40 & 5 & 1.30 & 5 \\
\hline 6 & 1.30 & 11 & 1.40 & 9 & 1.45 & 9 & 1.30 & 9 & 1.25 & 9 & 1.25 & 9 & 1.20 & 9 \\
\hline 7 & 1.85 & 9 & 1.50 & 8 & 1.37 & 8 & 1.37 & 8 & 1.32 & 7 & 1.32 & 8 & 1.30 & 8 \\
\hline 8 & 1.30 & 14 & 1.35 & 14 & 1.33 & 11 & 1.40 & 12 & 1.65 & 7 & 1.37 & 7 & 1.55 & 8 \\
\hline 9 & 1.40 & 10 & 1.45 & 10 & 1.40 & 11 & 1.37 & 10 & 1.35 & 10 & 1.35 & 9 & 1.30 & 8 \\
\hline Mean & 1.57 & 11 & 1.53 & 10 & 1.47 & 10 & 1.51 & 9 & 1.51 & 9 & 1.44 & 8 & 1.40 & 8 \\
\hline
\end{tabular}


pressure as well as under the normal pressure. Also the rate of heart beat showed only a slight change-a tendency to increase at the beginning of pressure increase, while that under the normal pressure rather decreased.

\section{Summary.}

Conductivity of perfusion fluid of frog heart was measured under abnormal atmospheric pressure during the perfusion.

The conductivity increased under high atmospheric pressure and decreased under low pressure.

Heart beats of frog showed no change of height under high atmospheric pressure but only a slight tendency to increase the rate at the beginning to the pressure increase. 